\title{
A review of some works in the theory of diskcyclic operators
}

\begin{abstract}
In this paper, we give a brief review concerning diskcyclic operators and then we provide some further characterizations of diskcyclic operators on separable Hilbert spaces. In particular, we show that if $\mathrm{x} \in \mathrm{H}$ has a disk orbit under $\mathrm{T}$ that is somewhere dense in $\mathrm{H}$, then the disk orbit of $\mathrm{x}$ under $\mathrm{T}$ need not be everywhere dense in $\mathrm{H}$. We also show that the inverse and the adjoint of a diskcyclic operator need not be diskcyclic. Moreover, we establish another diskcyclicity criterion and use it to find a necessary and sufficient condition for unilateral backward shifts that are diskcyclic operators. We show that a diskcyclic operator exists on a Hilbert space $H$ over the field of complex numbers if and only if $\operatorname{dim}(H)=1$ or $\operatorname{dim}(\mathrm{H})=\bigoplus$. Finally, we give a sufficient condition for the somewhere density disk orbit to be everywhere dense.
\end{abstract}

Keyword: Diskcyclic operators; Hypercyclic operators; Supercyclic operators; Weighted shift operators 